\title{
Entender las barreras a la innovación social que enfrentan los actores de la economía social y solidaria: un enfoque comunicacional
}

Nicolas Duracka*

Estudiante de doctorado, Laboratorio Communication et Solidarité, Universidad Blaise Pascal de Clermont-Ferrand, Aubière, Francia.

Correo electrónico:

nicolas.duracka@univ-bpclermont.fr

Recibido: 25 de agosto del 2015

Aceptado: 9 de noviembre del 2015

Cómo citar este artículo: Duracka, N. (2016). Entender las barreras a la innovación social que enfrentan los actores de la economía social y solidaria: Un enfoque comunicacional. Cooperativismo y Desarrollo, 24(108), 71-84. doi: http://dx.doi.org/10.16925/ co.v24i108.1261

\section{Resumen}

Propósito: este escrito propone un nuevo marco conceptual en tres dimensiones utilizando el componente ontológicamente interdisciplinar de las ciencias de la información y la comunicación. Descripción: propone un enfoque comunicacional de la innovación social de los actores de la economía social y solidaria, abordando la dinámica praxeológica de sentido común y la co-construcción de conocimiento compartido en un espacio público. Punto de vista: en particular, se cuestiona la dicotomía entre una identidad utilitaria y transformadora dentro de las organizaciones de la ess y las prácticas de comunicación que implica. Conclusiones: el avance descrito aquí tiene una tendencia para nosotros a desafiar las dicotomías establecidas en relación con la Ess (economía social isomorfa vs. economía solidaria transformadora), y la innovación social (una versión "débil” vs. una "versión fuerte"). De hecho, los procesos que se activan en estos dos campos son mucho más complejos y mucho más ambivalentes.

Palabras clave: barreras, comunicación, economía social y solidaria, innovación social. 


\title{
Understanding the Barriers to Social Innovation Faced by Social and Solidarity Economy Actors: A Communicational Approach
}

\begin{abstract}
Purpose: This article proposes a new conceptual framework in three dimensions, using the ontologically interdisciplinary component of Information and Communication Sciences. Description: It proposes a communicational approach to social innovation of social and solidarity economy actors, addressing the praxeological dynamics of common sense and the construction of shared knowledge in a public space. Point of view: In particular, the dichotomy between a utilitarian and transforming identity in sse organizations and the communication practices involved are questioned. Conclusions: The progress described herein has a tendency, in our opinion, to challenge the dichotomies established in connection with the sse (isomorphic social economy vs. transforming solidarity economy) and social innovation (a "weak" version vs. a "strong" version). In fact, the processes triggered in these two fields are much more complex and much more ambivalent.
\end{abstract}

Keywords: barriers, communication, social and solidarity economy, social innovation.

\section{Entender as barreiras para a inovação social que os atores da economia social e solidária enfrentam: um enfoque comunicacional}

\section{Resumo}

Propósito: este artigo propõe um novo referencial conceitual em três dimensões, utilizando o componente ontologicamente interdisciplinar das ciências da informação e da comunicação. Descrição: propõe um enfoque comunicacional da inovação social dos atores da economia social e solidária, abordando a dinâmica praxeológica de sentido comum e a coconstrução de conhecimento compartilhado num espaço público. Ponto de vista: em particular, questiona-se a dicotomia entre uma identidade utilitária e transformadora nas organizações da Ess e as práticas de comunicação que implica. Conclusões: o avanço descrito aqui tem uma tendência a desafiar as dicotomias estabelecidas com relação à Ess (economia social isomorfa versus economia solidária transformadora) e a inovação social (uma versão "fraca" versus uma versão "forte"). De fato, os processos que se ativam nesses dois campos são muito mais complexos e muito mais ambivalentes.

Palavras-chave: barreiras, comunicação, economia social e solidária, inovação social. 


\section{Introducción}

Si se trata de un término que ocupa cada vez más espacio en las diversas cuestiones académicas, y sobre todo, en las preocupaciones políticas contemporánea, es la innovación social. De hecho, la crisis en las sociedades occidentales durante décadas provoca un crecimiento exponencial de los movimientos sociales (por ejemplo, los Indignados en España). Como se destaca en tres grandes nombres francófonos, Juan Luis Klein, Jean Louis Laville y Franck Moulaert, la economía social y solidaria (ESs) es frecuentemente la base de estos movimientos para iniciar invenciones sociales que "constituyen la fuente de innovaciones sociales que puede transformar la sociedad y constituir la base de un modelo más democrático y participativo, pero puede igualmente consolidar el capitalismo en vigor $^{1}$ (Peck, 2013)" (Klein, Laville, y Moulaert, 2014, p. 9). Entre la transformación social y el isomorfismo institucional, nos centramos en el lugar de un enfoque comunicativo de la innovación social. En otras palabras, queremos cuestionar las prácticas de comunicación de los actores socialmente innovadores de la economía social y solidaria con el fin de informar la dicotomía entre la innovación "fuerte" y la innovación "débil" para explicar mejor las barreras específicas que encuentran estas iniciativas.

\section{Economía social y solidaria e innovación social: entre isomorfismo y transformación}

Detrás de la idea de innovación todavía cuelga esta noción de una ruptura radical con el pasado retrógrado. Pero hoy día la mayoría de los movimientos sociales llevan consigo una necesidad de transformación como un camino progresivo. Para informar sobre una concepción gradual para la transformación social, queremos movilizar las teorías del cambio institucional, incluyendo la mirada de Thorstein Veblen, que considera a la institución como "los hábitos mentales predominantes, formas generalizadas de pensar las relaciones y funciones particulares de la persona y la sociedad" (1970, p. 125). Estas instituciones son principalmente económicas, afectan los hábitos de pensamientos individuales y normalizan el comportamiento guiando la acción (hábitos de hacer), mientras que estructuran las relaciones sociales ${ }^{2}$. Para Veblen, el cambio institucional es naturalmente gradual porque está obstaculizado por las diferentes formas de conservadurismo. Así solamente puede ser considerado como un cambio a largo plazo y necesariamente progresivo (Besançon, 2014, p. 209) porque da lugar a prácticas seleccionadas adoptadas por los individuos y las organizaciones. Por lo tanto, en la sociedad contemporánea en gran medida incrustada en una lógica institucional dominada por la economía liberal, las acciones desarrolladas por la economía social y solidaria proponen cambiar estos hábitos y organizaciones colectivas que regulan al individuo. También propone "democratizar la economía a través de la participación ciudadana" (Laville, 1994). Sin embargo, es necesario distinguir dos conceptos:

1. La economía social, históricamente vinculada a los estatutos (asociaciones, cooperativas, mutualidades) y basada en el principio de "doble condición" de los accionistas (o miembros) y activos (o actores), según Draperi (2013). Las iniciativas sociales, fuertemente inspiradas en la corriente utópica (Fourier, Godin) y la corriente filantrópica (Le Play) sobre la que dicen que hay "otra manera de entrar en economía, que es confiar en la dimensión social de las personas y los valores que comparten" (Draperi, 2013, p. 30) lo que supone una fuerte dimensión comunitaria. Por último, es necesario tener en cuenta que la economía social se articula con "un movimiento social y un pensamiento [...] la economía social invita a cada actor social para convertirse en un investigador (y viceversa) en un modelo de investigación acción original" (Draperi, 2013, pp. 69-70). El individuo se convierte en un actor social sobre todo, lo que funda la libertad y la unidad de la persona.

2. Y la economía solidaria con la doble dimensión económica y política de las organizaciones que toman en cuenta el cambio institucional que se refiere a su relación con una solidaridad democrática (Besançon, 2014). De hecho, las iniciativas de solidaridad "no resultan solamente de los intereses comunes para un grupo, pero vienen de objetivos en relación con el modelo de sociedad" (Cary y Laville, 2015, p. 25). Por otra parte, los actores de la economía solidaria desean salir de una acción puramente socio-económica para invertir una doble dimensión económica y política. La política en 
el sentido de Lefort (1986), es decir, la cuestión de "vivir juntos" implica la democracia y las prácticas de deliberación colectiva. Esto significa que

Más allá del funcionamiento interno la posibilidad de influir en el cambio institucional depende de la aparición de espacios y foros a través de los cuales se hace concebible de influir sobre el equilibrio de poder lo que hace surgir en las alianzas apuestas como las de la co-construcción de políticas públicas" (Cary y Laville, 2015, p. 25).

Por último, esta dimensión política se basa en la pluralidad de los principios económicos que permiten salir de la falacia economista (Polanyi, 2007) y dar lugar a la redistribución y la reciprocidad (Polanyi, 1983).

El espacio no nos permite ir más allá y matizar nuestro propósito pero queremos subrayar el aspecto dicotómico de la Ess en su relación con el cambio institucional. De hecho, podemos ver que la economía social, a través de una dimensión política suplantada por el predominio de lo social, y por no cuestionar el principio económico, opera en una relación histórica de complementariedad con el mercado y el Estado. Entonces, este sector sufre de una tendencia isomórfica vista como un "proceso de unión que obliga a una unidad en una población a parecerse a otras unidades de esta población enfrentándose a la misma serie de condiciones ambientales" (DiMaggio y Powell, 1983, p. 149). Frente a la institución de la economía de mercado, la economía social a veces se convierte en una muleta para facilitar la retirada del Estado y la moralización del mercado. En otras palabras, el pionero movimiento obrero asociacionista ha sustituido su papel político por un papel gestor, cediendo gradualmente a la presión del mercado, en gran parte legitimada por el Estado. Por lo tanto:

La lógica de la reacción a los efectos del capitalismo, lo que explica la apariencia de las organizaciones de la economía social, se atenúa a favor de una lógica de adaptación funcional a este modo de producción que se combina con el mantenimiento de características notables, sin embargo eso se traduce sobretodo en su estructura interna (Laville, 1994).

Por otra parte, las iniciativas de solidaridad ligadas a una ruptura con los principios del mercado económico y una reafirmación de la dimensión política del compromiso colectivo en el ámbito público, tienden en general a una voluntad de cambio institucional. Por supuesto, esta dicotomía tiene muchos ejemplos contrarios, pero una tendencia general observable desde hace décadas en la economía social tiende a validar esta inflexión.

Muchos autores consideran que la economía social es una "marga fértil para la innovación social" (Besançon, Chochoy y Guyon, 2013; Bouchard, 2011; Lévesque, 2007; Lévesque, 2002). Esta analogía se basa en su dimensión ontológicamente colectiva (Hiez y Lavillunière, 2013) y democrática como un vector de transformación social. También con la voluntad de la ess de promulgar otras formas de coordinación y cooperación que las relaciones de mercado e incluso participar en un proceso de re-incorporación de la economía en un proyecto político democrático (Dacheux, 2011a; Laville, 2011).

Ampliamente fantaseado por el espejismo de Schumpeter (1939), que ha posicionado durante mucho tiempo el progreso técnico en el corazón de la economía, hoy día:

El mito del progreso continuo en relación con el cambio técnico es parcialmente roto. Así, no es por casualidad que el tema de la innovación social está tomando un lugar cada vez más importante en las preocupaciones de las organizaciones, tanto públicas como privadas (Durance, 2011, p. 6)

Que la innovación sea tecnológica o social es sobre todo un proceso que hace referencia a un contexto social emergente "del cual el territorio es uno de los componentes" (Richez-Battesti, 2008, p. 21). Sin embargo, la dimensión social de todas las innovaciones todavía toma cuerpo en "el establecimiento de nuevas prácticas o la mejora de las prácticas existentes" (Besançon, Chochoy y Guyon, 2013, p. 23) acerca de la innovación social. Por tanto, se refiere esencialmente a los "modos de hacer" (Cloutier, 2003) lo que le da una genética profundamente inmaterial. Por otra parte, si la innovación tecnológica está incrustada en una doble lógica de la economía de mercado y de la gestión de personas (enlace descendente y lógica de management), la innovación social implica a su vez "la inversión de la forma tradicionalmente aceptada para llevar antes los propios individuos" (Besançon, Chochoy y Guyon, 2013, p. 22), en un doble sentido de la economía social y solidaria (revalorización de la cooperación y la reciprocidad) y la deliberación (proceso democrático ascendente). De hecho, la innovación social aparece 
en la literatura en los años sesenta mediante la designación de "nuevas formas de hacer las cosas con el propósito explícito de dar respuesta a las necesidades sociales" (Taylor, 1970, p. 70). Se verá rápidamente como un proceso social que involucrará actores heterogéneos y facilitará el empoderamiento (Chambon, David y Devevey, 1982; Cloutier, 2003). Este concepto será finalmente utilizado en el ámbito institucional por el Manual de Oslo en 1997 y en el 2005, cuando el término aparece en la propia innovación social (OCDE, 2005, p. 54). Hay que tener en cuenta, sin embargo, que la dimensión social aparece especialmente en las prácticas de la organización (empresa) y no vuelve, o poco, a la sociedad civil. Por último, los investigadores del Instituto Godin (Francia) se acercan al debate contemporáneo de la innovación social proponiendo una tipología que combina dos conceptos y cuatro enfoques (Besançon, Chochoy y Guyon, 2013).

1. El primer diseño es (más bien) anglosajón, de carácter individual, y da importancia a la novedad y la finalidad social.

- Incluye un enfoque por la modernización de las políticas públicas con perspectivas a la competitividad y al atractivo de los territorios en una lógica de instrumentalización y retirada de las políticas públicas.

- Pero también hay un enfoque por el emprendimiento social siguiendo las grandes universidades de los Estados Unidos y los círculos empresariales para "moralizar" la figura del empresario (por ejemplo, Ashoka).

2. El segundo diseño es de naturaleza colectiva y se centra en el proceso de innovación social.

- Nos encontramos con el enfoque de la empresa social (e. g. la red emes en Europa), que nota la importancia de un proceso de abajo hacia arriba sobre la base de los principios democráticos y de carácter no lucrativo. Este enfoque se basa en la respuesta a las necesidades de la comunidad que se encuentra en el corazón de la economía social.

- Por último, nos encontramos con un enfoque institucionalista (enfoque muy desarrollado por el CRISES ${ }^{3}$ ) en el que la innovación social es

Centro de Investigación Sobre las Innovaciones Sociales en Economía Social. una aspiración social, un deseo de cambio, que se concreta en un proceso colectivo, basado en fuertes raíces locales y una forma de organización democrática. La novedad aparece en las prácticas de ruptura con las prácticas habituales en un contexto determinado (Besançon, Chochoy y Guyon, 2013). Este último se basa en gran parte en las iniciativas de economía solidaria.

Podemos ver que la economía social y solidaria está sufriendo una tensión entre una tendencia isomórfica (ampliamente atribuida a las iniciativas sociales) y una tendencia a profundizar el cambio institucional (más ampliamente reconocible en las iniciativas solidarias). Además, en cuanto a la tipología teórica propuesta por los investigadores del Instituto Godin, es posible decir que los dos primeros enfoques de la innovación social tienen una dimensión "débil" porque intentan responder a las necesidades sociales con una lógica utilitarista (respuesta a la retirada del Estado y de las empresas sociales). Al contrario, los dos últimos enfoques son de tipo "fuerte" porque se basan en una lógica de transformación democrática y social (la lógica de la cooperación y la ruptura contextualizada). Entre isomorfismo y transformación social, tenemos una primera observación que podemos hacer y que permite, en primer lugar, comprender por qué los actores de la economía social están experimentando problemas de comunicación (Dacheux, 2007; Dacheux, 2011b). En segundo lugar, este descubrimiento nos permite emitir una nueva hipótesis. Todavía con la idea de que la ess es un laboratorio de innovación social, opinamos que los actores de la innovación social también tienen barreras de comunicación. Pero, ¿de qué tipo de comunicación estamos hablando?

\section{Entre la comunicación política y el marketing político, se centran las prácticas comunicativas de la economía social y solidaria}

Aquí nos preocupan las organizaciones que agrupan "todas las actividades que contribuyen a la democratización de la economía a partir de compromisos sociales" (Laville y Cattani, 2005). Pero estas actividades no se limitan a los registros de asociados y de comunidad porque se inscriben en el principio de asociación cuya originalidad consiste en "ser parte de un espacio democrático para exigir la libertad y la igualdad entre sus miembros. Se está profundizando la reflexión sobre la 
democracia que hace posible entender el hecho asociativo" (Laville y Sainsaulieu, 2013, p. 258). Estas organizaciones son actores principalmente políticos que por lo tanto constituyen la institución de un espacio público que se diferencia de otras comunidades. Entonces, debería lógicamente desarrollar una forma adaptada de comunicación. Por lo tanto, "de manera intuitiva, la comunicación política evoca todo con respecto a la producción y al intercambio de discursos políticos realizados por los diversos actores y representados por los medios de comunicación" (Wolton, 1989, p. 28). Más específicamente, la comunicación política, como cualquier comunicación, es profundamente ambivalente (Dacheux, 2004), pero está principalmente usando dos caminos separados. Una primera dimensión normativa que hace referencia a la teoría de la acción comunicativa de Jürgen Habermas (1987) que se refiere al sentido compartido de un "mundo vivido", que:

Constituye el horizonte de los juicios de intercomprensión mutua en la que los participantes (de un acto de comunicación) entran en acuerdo o en oposición acerca de algo que pertenece a la singularidad del mundo objetivo, la particularidad del mundo subjetivo, o a la comunidad del mundo social (p. 148).

Esta dimensión de la comunicación política es vista como dialógica articulando la publicidad política como transmisión de información política para la mayoría, de manera que cada ciudadano puede hacerse a una opinión racional, y al diálogo racional, como toma de palabra en público entre las personas que apriori disfrutan de una igualdad de competencia y que aceptan los procedimientos para el buen desarrollo de la discusión cuyo objetivo es la búsqueda de un consenso racional. Esta dimensión normativa de la comunicación política constituye así un horizonte ideal que raramente se logra. De hecho, se agrega la mayoría de las veces a una dimensión estratégica de la comunicación que consiste en enviar un mensaje fuerte a un receptor más o menos activo con el fin de superar sus puntos de vista. Detrás de esta forma estratégica de la comunicación política se encuentra la construcción y el uso de símbolos políticos (bandera, lema, himno, etc.) para reforzar la cohesión de la comunidad política. También se esconde detrás de la comunicación estratégica, la negociación política que se caracteriza por el hecho de que todo el mundo se comunica para maximizar sus intereses, mientras se busca llegar a un compromiso. Y finalmente, la persuasión política que consiste en agregar más personas a su campaña. En el pasado, la comunicación política se tomó los caminos de la retórica, ahora, principalmente a través del uso de técnicas del marketing, se toma más y más el camino de la "comunicación marketing".

De hecho, hemos observado empíricamente:

Que las empresas, las asociaciones, las instituciones políticas utilizan los mismos medios de comunicación en los espacios públicos [...] Estas estructuras, asociaciones como instituciones, se ven tentadas por unas comunicaciones corporativas y niegan lo que es su fuerza, es decir, una comunicación política ${ }^{4}(\mathrm{Di}-$ lliere-Brooks, 2007).

La también llamada "comunicación marketing" se refiere a "todas las señales emitidas por la empresa hacia sus diferentes públicos, es decir a sus clientes, distribuidores, proveedores, accionistas, con las autoridades públicas y también hasta su propio personal" (Decaudin, 1999, p. 11). Una forma de comunicación principalmente persuasiva que consiste en transmitir un mensaje a las audiencias para alterar el comportamiento mental (Lindon, 1991) cuyos fundamentos se apoyan en un modelo llamado "telégrafo" de la comunicación (Shannon y Weaver, 1975) o el envío de un mensaje desde un transmisor hacia un receptor. La comunicación marketing también responde a dos trayectorias distintas. La primera se refiere al marketing operativo que incluye la planificación, el control y la organización del departamento de marketing. La segunda se refiere a un marketing estratégico conformado por tres pasos:

- Se trata, en primer lugar, de segmentar una población a través de un "corte del mercado en subgrupos de consumidores con características comunes que pueden explicar las diferencias de comportamiento de los subgrupos homogéneos así constituidos" (Chirouze, 2007, p. 31).

- El siguiente es el posicionamiento de marca que es "una respuesta estratégica a la congestión de los mercados y que consiste a dar a un producto una posición específica en la mente de los consumidores para que se destaquen de los productos de la competencia" (Chirouze, 2007, p. 337).

- Fijar los objetivos de marketing de segmentos seleccionados.

Entrevista con Eric Dacheux, especialista francés de las ciencias de la información y de la comunicación. 
- Edificar el "marketing mix" o "la elección, la dosis y la combinación de los medios con los que la empresa quiere influir en el mercado" (Chirouze, 2007, p. 377).

Este último paso es formar un todo sinérgico entre la suma de las operaciones ${ }^{5}$ de promoción que forman la parte visible del iceberg y que los especialistas del marketing llaman "comunicación", cuando los críticos lo llaman "com", y que preferimos llamar “comunicación marketing” (Décaudin 1999). Este último, se entenderá que es puramente estratégico, se trata de persuadir y de aumentar los beneficios de la organización. Tiene sus raíces en una tecnología intelectual que tiene un objetivo lucrativo y se alimenta de investigaciones con una concepción utilitarista del conocimiento. Es una herramienta que nació en el mundo de la empresa y que consiste en desarrollar una combinación coherente de diferentes medios de acción para alcanzar los objetivos en un contexto competitivo.

Sin embargo, esta técnica ha sido apropiada por el campo político. De hecho, en 1932, Franklin Roosevelt aprovecha el programa de radio Fireside Chats ("Charlas al Amor de la Lumbre") para enviar su mensaje a los ciudadanos estadounidenses. Seguirá la creación de empresas de comunicación y la creación de técnica de marketing político. Esto permite al político, o a la institución política que lo utiliza, reunir información acerca de la población, para segmentarla de forma homogénea y luego definir una imagen, un discurso que le asegura un posicionamiento lo más favorable posible en los segmentos más rentables. El marketing político es una metodología cuyo objetivo es adaptar la oferta política a la demanda social, persuadir a los ciudadanos de que las propuestas del candidato, o la institución, corresponden a las expectativas explícitas o implícitas que los especialistas habían creído detectar. El objetivo del marketing político es tener la adhesión de un blanco a un proyecto diseñado por otro. Además, el marketing comercial está profundamente opuesto a la esencia de la política en su intento de separar la articulación para establecer objetivos específicos, por lo que se opone al proyecto democrático de la diversidad, de unirse para construir un fin común que no es único y universal a la imagen del consumidor ideal.

Por último, hay que tener en cuenta que la comunicación, ya sea de marketing o política se desarrolla en las organizaciones en una doble dimensión

Tabla 1

Comparación entre la comunicación marketing (Decaudin, 1999) y la comunicación política (Wolton, 1989)

\begin{tabular}{|c|c|c|c|}
\hline & & Comunicación marketing & Comunicación política \\
\hline Organizaciones internas & & $\begin{array}{l}\text { - Servicios (marketing, } \mathrm{RH} \text { ) } \\
\text { - Jerárquico } \\
\text { - Por proyecto }\end{array}$ & $\begin{array}{l}\text { - Espacio público de proximidad } \\
\text { - Democrático } \\
\text { - Horizontal }\end{array}$ \\
\hline \multirow[b]{2}{*}{ Objetivos } & Interna & $\begin{array}{l}\text { - Gestión de recursos humanos } \\
\text { - El empleado tiene que adherirse }\end{array}$ & - Crear una identidad compartida \\
\hline & Externa & $\begin{array}{l}\text { - Aumentar la cuota de mercado } \\
\text { - Aumentar volúmenes de ventas } \\
\text { - Contribución con beneficios empresariales }\end{array}$ & $\begin{array}{l}\text { - Favorecer el debate } \\
\text { - Sin fin de lucro }\end{array}$ \\
\hline \multirow[b]{2}{*}{ Actores implicados } & Interna & - Empleador & - Ciudadanos libres a participar \\
\hline & Externa & $\begin{array}{l}\text { - Consumidores } \\
\text { - Blancos determinados por segmentación del } \\
\text { mercado }\end{array}$ & $\begin{array}{l}\text { - Ciudadanos que participan al debate } \\
\text { público }\end{array}$ \\
\hline \multirow[b]{2}{*}{ Herramientas } & Interna & $\begin{array}{l}\text { - Seminarios } \\
\text { - Intranet }\end{array}$ & - Deliberación \\
\hline & Externa & - Mix de comunicación marketing & $\begin{array}{l}\text { - Concertación } \\
\text { - Talleres colectivos } \\
\text { - Reuniones públicas }\end{array}$ \\
\hline
\end{tabular}

Nota. Elaboración propia.

$5 \quad$ Publicidad de medios, publicidad directa, la publicidad por evento, relaciones públicas, diseño, embalaje, fuerza de ventas, lobbying, el boca a boca, ferias y exposiciones (Decaudin, 1999). 
(tabla 1). En primer lugar, en una dimensión interna que actúa, en el caso marketing, en el corazón de los servicios (marketing o recursos humanos) buscando la adhesión de los empleados a la estrategia de la empresa a través de herramientas como seminarios o intranets (cartas o informaciones empresariales). En el caso de una comunicación política, se refiere más a una dimensión como parte de un espacio público de proximidad, con objetivo al establecimiento de una identidad compartida entre los miembros libres, unidos a través de un proceso de deliberación. En segundo lugar, una dimensión externa (en el caso del marketing) viene a perseguir los objetivos desarrollados internamente y a aumentar las cuotas de mercado, volúmenes de ventas y contribución a los beneficios de la empresa, apuntando a los consumidores "blanco" a través del mix marketing. Al contrario, se intentará favorecer el debate en el espacio público a través de consultas públicas e intercambios para las organizaciones de la Ess sin fines de lucro.

Desde la mirada centrada en la dinámica del cambio institucional en el sentido de Veblen (1970), se destacan sucesivamente las dimensiones dicotómicas de la economía social y solidaria, de la innovación social y de la comunicación que se encuentran en la literatura. Ahora me gustaría enfrentar este marco teórico en mi campo de estudio, a la región de Auvergne, Francia. Voy a empezar con un breve desvío metodológico que nos permitirá identificar las dimensiones "débiles" y "fuertes" de los proyectos estudiados con el fin de analizar las prácticas de comunicación posteriores.

\section{Un método exploratorio}

En primer lugar, me gustaría explicar un marco metodológico que nos sitúa en un enfoque reflexivo, proponiendo, para empezar, nuestros "supuestos normativos" (Burawoy, 2003) y pugnando "a favor de un compromiso en el mundo a través de la racionalidad de la teoría" (Polanyi, 1974). Lo que nos obliga a recordar que el autor de estas líneas es principalmente una persona comprometida en la Ess y la innovación social, y que es una gran oportunidad porque el "trabajo de campo depende de cabo a rabo de la capacidad del investigador para ser deportado al punto de vista de los demás" (Céfai, 2003, p. 517). Luego, con el fin de explorar la hipótesis de trabajo, se decidió llevar a cabo un proceso exploratorio en cuatro pasos (figura 1):

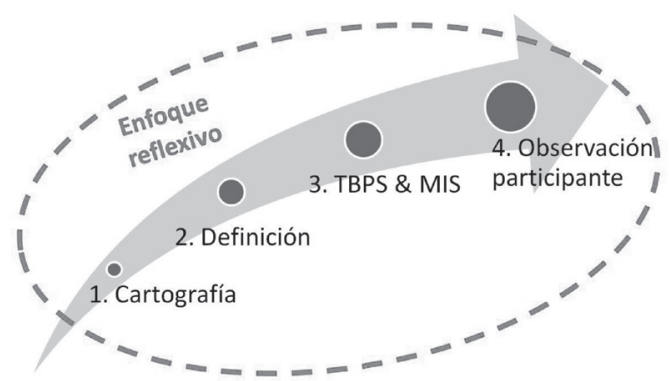

Figura 1. Las cuatro etapas del enfoque metodológico. Elaboración propia.

1. Cartografía de los actores de la economía social y solidaria en la región de Auvergne (contexto de estudio territorial). Entonces, decidí segmentar todos los actores heterogéneos del territorio en dos grandes categorías. Los primeros, llamados "actores principales", incluyen a todos los actores que se reclaman explícitamente (por sus estatutos, sus nombres, sus funciones o prácticas) del mundo de la ess. El segundo incluye los "actores secundarios" o grupos de interés que se convierten solo en parte, sin notificar al público, a la ess.

2. Entrevistas semiestructuradas con actores clave, con base en una premisa teórica considerando la comunicación como una actividad de construcción de sentido compartido y de comprensión mutua basada en la alteridad y en la interpretación que para funcionar, tiende a desarrollar una base de "conocimientos normativos compartidos" (Mucchielli, 2000). Así he entrevistado a los "actores principales" del territorio para conocer :

- Su definición de la economía social y solidaria.

- Su definición de la innovación social.

- Una lista de proyectos de innovación social del territorio que corresponden a sus criterios (para el paso 3).

Se ha propuesto una lista de 33 proyectos, de los cuales tres han sido especialmente citados:

- La scic ${ }^{6}$ Combrailles Durables, que tiene como objeto principal el desarrollo de actividades

\footnotetext{
6 Sociedad Cooperativa de Interés Colectivo. Tiene la particularidad de funcionar como una cooperativa pero con la posibilidad de tener una colectividad pública dentro del consejo de administración y del capital.
} 
con carácter social y solidario en el contexto del desarrollo sostenible. En otras palabras, desde diciembre del 2008, más de 200 colaboradores han desarrollado un enfoque de cooperación que incluye la producción de "energía ciudadana" a través de las centrales fotovoltaicas instaladas en los techos de las comunidades del territorio rural. Todos los actores de la scic son rurales, voluntarios y tienen un estatus social y profesional que les da un fuerte capital socio-cultural.

- La asociación Epicentre-Cowork, que tiene como base de trabajo la puesta en común de un lugar de trabajo como un portador de un proyecto de colaboración de los actores económicos heterogéneos. Es un espacio de coworking o "tercer lugar creativo de economía solidaria" que existe desde julio de 2013 y está siendo ampliado para dar espacio a un "anti-café" y un FabLab. ${ }^{8}$

- La asociación JeRecycleParc, con el objetivo, desde octubre del 2013, de desarrollar una ressourcerie y revitalizar el barrio (zona de la estación). La actividad de la ressourcerie se centra en la recogida (con individuos particulares), la transformación y la reventa de objetos (ropa, libros, artículos para el hogar, etcétera). Para favorecer el surgimiento de comportamientos eco-ciudadanos y mejorar la calidad de vida de su territorio, se desarrollan servicios de proximidad creadores de vínculos sociales y actividades económicas innovadoras, con un enfoque específico en las actividades de reutilización. Los actores de la ressourcerie son en su mayoría procedentes de squat. ${ }^{9}$

3. El tercer paso de la metodología de estudio es una evaluación de la solidaridad y las prácticas innovadoras de estos tres proyectos, basado en el trabajo del Instituto Godin (Besançon y Chochoy, 2014; Besançon, 2014). El principal objetivo de este paso es validar la presencia en el territorio de. estudio de proyectos basados en prácticas llamadas "utilitaristas/débiles" o "transformadoras/fuertes". Por tanto, los resultados muestran al menos dos características diferentes (véase tabla 2).

El café se paga a la hora.

8 Lugar de transformación de objetos sobre todo electrónicos.

9 Lugares ocupados ilegalmente.
Tabla 2

Características de los tres proyectos examinados por los cuadros de mando de las prácticas solidarias y los marcadores de innovación social (Instituto Godin)

\begin{tabular}{ll}
\hline & \multicolumn{1}{c}{ Características } \\
\hline $\begin{array}{l}\text { Asociación Epi- } \\
\text { centre-Cowork }\end{array}$ & $\begin{array}{l}\text { Economía colaborativa. Aspira a la creación } \\
\text { de riqueza por el intercambio (coworking). } \\
\text { El proyecto tiene por objeto la respuesta a } \\
\text { las necesidades. Ausencia de un espacio de } \\
\text { debate público. }\end{array}$ \\
\hline & $\begin{array}{l}\text { Economía circular. Aspira a la creación de } \\
\text { una dinámica de reciprocidad generadora } \\
\text { de vínculo social. Lugar de generación de } \\
\text { debate sobre la cuestión del re-empleo de los } \\
\text { productos de consumación. El proyecto está } \\
\text { en ruptura contextualizada con el existente. }\end{array}$ \\
\hline Recycle Parc & $\begin{array}{l}\text { Economía cooperativa. Aspira a la trans- } \\
\text { formación social a propósito del tema de la } \\
\text { energía. Proyecto sostenido por un colectivo } \\
\text { de voluntarios que tiene un capital social y } \\
\text { cultural fuerte. }\end{array}$ \\
\hline lles Durables &
\end{tabular}

Nota. Elaboración propia.

4. Para finalizar, se realizaron tres estudios de caso sobre las prácticas de comunicación dentro de los proyectos.

\section{Las prácticas de comunicación de los actores de la innovación social: estudio de caso}

A través del enfoque metodológico, se observó que el proyecto Epicentre-Cowork tiene ciertas características específicas del emprendimiento social dándole una dimensión "débil". Al contrario, la asociación JeRecycleParc tiene una fuerte aspiración a la ruptura y ciertas prácticas genéticamente "fuertes". Esta observación también aplica para la scic Combrailles Durables sin poder todavía distinguirla de la precedente. Por lo tanto, en referencia a la dicotomía comunicativa presentada anteriormente, se quiere saber si las prácticas de comunicación política están implementadas por los actores de la innovación "fuerte", o si los actores de la innovación social "débil" movilizan las prácticas de comunicación del marketing.

Para ir en la dirección del paso metodológico anterior, es necesario proponer una evaluación de las prácticas de comunicación. Entonces, se opta por un enfoque praxeológico que permite poner de relieve la comunicación entre los actores como "actividad 
organizadora" que tiende a construir "una perspectiva común y refleja principalmente procesos de organización de una visión compartida" (Quéré, 1991, p. 76). En otras palabras, si las acciones solidarias permiten aclarar lo que hace una organización, las prácticas de comunicación tratarán de describir cómo lo hace. Así, estas se refieren a las prácticas que dan cuenta de la constitución de una comunidad de lenguaje (concedemos un lugar esencial al lenguaje para entender la construcción de la realidad) y la fabricación de las mediaciones simbólicas compartidas (conceptos, representaciones, de materialización). Las prácticas de comunicación son actividades conjuntas para construir una perspectiva común, una visión compartida (Quéré, 1991) a partir de la conformación mutua de un mundo común a través de una acción combinada (Varela, 1989). Por lo tanto, se han seleccionado algunas de las prácticas de comunicación importantes para cada una de las estructuras estudiadas.

Para empezar, la asociación Epicentre lleva una forma de innovación social "débil" que parece insertada en las prácticas de comunicación marketing. De hecho, de las observaciones se conservan dos elementos principales que llevan a esta conclusión. En primer lugar, la comunicación interna es regulada por algún tipo de liderazgo asumido por los dos co-fundadores del proyecto. Uno de los coworkers resume la situación así: "En el grupo hay proactivos. Si hay algo que hacer no necesariamente esperan que la decisión colectiva sea tomada, lo hacen. $\mathrm{Y}$ hay reactivos, es decir, las personas que esperan y siguen". Esta regulación por la impetuosidad está basada en la dependencia de los "líderes" que a veces toman decisiones sin consultar los otros miembros y representan la estructura al exterior sin necesariamente transmitir todas las informaciones. Se puede encontrar una explicación a este fenómeno en la identidad del proyecto de coworking. De hecho, la mayoría de los compañeros de trabajo están buscando una alternativa a la empresa convencional sin estar totalmente separada porque algunos siguen trabajando para una compañía que todavía tiene un funcionamiento gerencial y jerárquico (por ejemplo, el teletrabajo), mientras que algunos encuentran la independencia y están siempre en busca de la relación jerárquica que tranquiliza (empresarios autónomos neo-rural o independiente). Así que hay una verdadera paradoja entre el discurso de la economía colaborativa y la realidad de las prácticas que un integrante trata así: “YYo no soy utópico como para decir que se necesita ser participativo y democrático al 100\%!". Luego, la comunicación externa se realiza principalmente a través de la presencia en el campo de las relaciones públicas de los dos co-fundadores portadores del "liderazgo", pero también a través de la utilización recurrente de la publicidad por evento. De hecho, en colaboración con las organizaciones institucionales $\mathrm{u}$ organizaciones privadas, el espacio de coworking será a veces el teatro de eventos mediatizados y del encuentro con actores institucionalizados (e. g. "Up'hero" en colaboración con la marca territorial "Auvergne Nuevo Mundo", el proyecto "Shaker" con la cámara regional de la Ess, el "Ulule tour", entre otros).

Por otra parte, como hemos visto anteriormente, la asociación JeRecycleParc lleva una identidad transformadora pero vamos a ver que las prácticas de comunicación son mucho más ambivalentes. Internamente, la organización se estructura en torno a un "espacio público de proximidad" (Eme y Laville, 1992) en el que se comparten y se regularizan las prácticas deliberativas (encuentros semanales y consejo todos los meses). Estas prácticas se refieren a una dimensión normativa de la comunicación política apoyando una idea de habilidad conjunta (Frère, 2006) y cediendo a los procedimientos para la buena marcha de la discusión que tiene como objetivo la búsqueda de un consenso racional. Me encuentro con la explicación de la horizontalidad intrínseca al proyecto a través del concepto de "mundo vivido compartido" (Habermas, 1987). En la teoría de la acción comunicativa, Habermas (1987) sostiene que "el resultado de la interacción depende de la capacidad de los participantes para escucharse mutuamente sobre una evaluación intersubjetivamente válida de su relación con el mundo" (p. 122). Lo esencial de este modelo es el consenso entre los participantes, y de hecho, la gran mayoría de los actores del proyecto han vivido en el mundo squat y comparten muchos códigos y valores que crean un mundo común y facilitan la participación en la deliberación conjunta. Por otra parte, esta identidad en términos de comunicación externa ya tiene importancia. Así, de inmediato JeRecycleParc se estableció como actor político en el territorio empezando a amenazar a la comunidad de crear un squat si un local no se lo ofrecía a ellos. Vemos que los actores mezclan una dimensión puramente normativa de comunicación con una dirección estratégica para difundir sus aspiraciones colectivas. En otras palabras, ellos desarrollan una forma normativa para la publicidad política (flujo de información) y el diálogo (hablando en el espacio público a través de presencias en la acción colectiva cívica). Pero también usan una 
forma estratégica con el simbolismo del squat para activar un poder de negociación y maximizar sus intereses al intentar establecer un compromiso. Vemos surgir una forma de "comunicación persuasiva", definida como una "voluntad humana para establecer relaciones sociales pacíficas cuyo principal objetivo es provocar un cambio en el pensamiento o el comportamiento de los demás" (Dacheux, 1994, p. 29). En esta definición, la palabra "voluntad" indica la distinción necesaria entre la influencia y la persuasión. Cualquier comunicación, lo sabemos por el trabajo de la escuela de Palo Alto, genera una influencia. Pero es importante notar la diferencia de naturaleza entre el deseo de influir y la que ejercemos de manera inconsciente. La primera, por tanto, se refiere a una forma de "comunicación persuasiva" que tiene una intención estratégica, un "acción estratégica" diría Habermas (1987), que se distingue de la segunda, la "acción comunicativa", que se refiere más a una forma de "comunicación política”.

Por último, y a primera vista, la genética transformadora de la scic Combrailles Durables parece llevarla a desarrollar prácticas comunicativas similares a la estructura estudiada previamente. Primero, una regulación deliberativa y horizontal de la comunicación interna que también apoya la formación de un espacio público de proximidad. Uno de los miembros lo explica así:

Cuando se enojan es nunca malicioso o insalubre porque tienen diferencias de perspectivas, que defienden, pero al final ellos tienen la capacidad de decir que no tomemos la decisión esta noche y vamos a enriquecernos de otros puntos de vista, o decir, bueno, escucho, no estoy de acuerdo contigo, pero lo dejo esta vez porque sobre otros puntos vas a tener que dejarlo tú. Así hay un verdadero valor de la escucha, del compromiso, pero en la búsqueda de sentido, sin compromiso, pero la idea es enriquecerse de la opinión del otro.

En segundo lugar, si la dimensión estratégica se muestra claramente con el proyecto JeRecycleParc activando una forma consciente de comunicación persuasiva (amenaza de "squat"), se hace más complejo para este caso. De hecho, Combrailles Durables conserva una dimensión normativa (la publicidad y el diálogo), pero el uso de la forma de comunicación persuasiva (causar un cambio en el pensamiento o en el comportamiento de los demás) adquiere otra dimensión porque es inclusiva y mucho más difusa. En otras palabras, los integrantes de la scic no utilizan las amenazas o la publicidad directa (para el evento o los medios de comunicación), pero usan la negociación para maximizar su interés (el cambio que se produzca en la descentralización de la cuestión de la energía en Francia) con una estrategia menos consciente pero mucho más "velada" mediante la integración de las autoridades locales en sus órganos decisionales (Estatus SCIC), y se introducían en los lugares de las decisiones políticas ${ }^{10}$. Encontramos una explicación a esta estrategia más difusa. En las palabras de uno de los miembros "una cosa divertida en Combrailles Durables es la procedencia de la gente de diferentes orígenes, diferentes profesiones, diferentes edades porque no estamos lejos de tres generaciones... pero produce una alquimia increíble". Entonces, esta heterogeneidad del colectivo se convierte en una fuerza porque se apoya en personas que tienen un capital social y cultural muy alto (un profesor, ingenieros, responsable financiero de una empresa del CAC40, entre otros). Por lo tanto, comparte la voluntad de transformación (descentralización de la cuestión de la energía en Francia y reapropiación ciudadana), pero tienen identidades individuales que facilitan la creación de una identidad colectiva, porque las personas no buscan consolidar su identidad personal, ya que están ampliamente satisfechos con sus vidas personales y profesionales. Más allá de la implicación de esta constatación sobre la regulación interna, también se facilita la comunicación externa a través la normatividad aparente de los actores. Estos están "velados" detrás del compartimiento "aparente" de los "hábitos de hacer y de pensar" de la institución social, lo que facilita enormemente la comunicación y reduce las barreras.

\section{Barreras de comunicación actores de la innovación social: a modo de conclusión}

Se puede confirmar que existen diferentes prácticas de comunicación con respecto a la identidad de los proyectos. Sin embargo, la voluntad de ruptura endógena al cambio institucional (Veblen, 1970) que llevan las iniciativas de innovación "fuerte", a través de las prácticas de comunicación política, parece encontrar obstáculos distintos a los que se enfrentan los innovadores "débiles".

10 Uno de los integrantes está elegido en una institución regional. 
Los actores de la asociación de coworking tienen un obstáculo obvio, como resultado directo de la tendencia al liderazgo, y es la dificultad de crear un grupo unido compartiendo una identidad común a través de un compromiso. El colectivo de personas asociadas es heterogéneo, por lo tanto, tiene dificultades para forjarse, y tomará algún tiempo hacerlo, si tiene un verdadero deseo de alejarse del modelo "gerencial". Esta dificultad es aún más importante, en comparación con que estas prácticas internas faciliten en gran medida la comunicación externa. De hecho, los líderes se convierten en interlocutores casi únicos, incluidos los actores institucionales que parecen mucho menos perturbados por la verticalidad de la organización (oponiéndose a la horizontalidad de un grupo sin líder). Por último, la identidad del proyecto, aunque no se asume totalmente como tal, está directamente relacionada con el emprendimiento social y la economía colaborativa. Esta forma económica que está de moda hoy día en un modelo europeo en crisis, finalmente, no presenta una profunda transformación. Ahora, todos sabemos que el cambio genera un sentimiento de inseguridad, y una iniciativa original, pero poco transformadora es menos perjudicial para el orden institucional y por lo tanto más aceptable, lo que facilita enormemente la comunicación del proyecto.

Esta conclusión es totalmente revertida cuando nos fijamos en las barreras de comunicación de la ressourcerie. De hecho, estos actores tienen un "mundo vivido compartido" (Habermas, 1985) que facilita la co-construcción de una identidad colectiva. Esto muestra claramente una ruptura de las prácticas y formas de pensar en el contexto territorial. Se trata de una innovación "fuerte" que se hace mucho más difícil de soportar, con el objetivo de cambio institucional. La distancia entre la visión del mundo de un integrante de la ressourcerie, destacados del mundo squat, y la visión de un ciudadano medio o de un actor político, es muy importante y difícil de llenar. Es por eso que los propios integrantes hablan de "utopía pirata", es decir, una forma de utopía colectiva que fluye "al abordaje" de la institución social. Así que ella utiliza una práctica comunicativa como el chantaje para "abordar" la comunidad y imponer su lugar en un espacio público local.

Por último, la scic tiene un perfil similar a la ressourcerie con su capacidad de generar una utopía compartida dentro de un grupo cuyos "mundos vividos" están relativamente cerca, por lo menos, por su visión de la cuestión de la energía en Francia. Pero este grupo de individuos heterogéneos no moviliza, por su parte, una estrategia de "abordaje" consciente para encontrar un lugar en el espacio público. No, gracias a un capital sociocultural alto, los actores de la scic expulsan el obstáculo y se basan en el intercambio de una base normativa con la mayoría de los actores del conservadurismo institucional para interferir en el corazón del espacio público, "oculto" detrás de un aparente cumplimiento de hábitos de pensamiento y hábitos de hacer de las instituciones sociales. Sin embargo, es importante señalar que esta postura expone estos "velados" al riesgo constante de deslizamiento esquizofrénico entre un

Tabla 3

Prácticas de comunicación de las organizaciones estudiadas

\begin{tabular}{|c|c|c|c|}
\hline & Comunicación interna & Comunicación externa & Obstáculos/2. Facilidades \\
\hline $\begin{array}{l}\text { Epicentre Cowork } \\
\text { "débil" }\end{array}$ & $\begin{array}{l}\text { De gestión } \\
\text { Figura del líder }\end{array}$ & $\begin{array}{l}\text { Marketing } \\
\text { Publicidad por el evento }\end{array}$ & $\begin{array}{l}\text { Disminución de la dinámica } \\
\text { interna del grupo. } \\
\text { Se comparte una base normativa } \\
\text { institucional }\end{array}$ \\
\hline $\begin{array}{l}\text { JeRecycleParc } \\
\text { "fuerte" }\end{array}$ & $\begin{array}{l}\text { Deliberativa } \\
\text { Encuentros cotidianos } \\
\text { Utopía colectiva }\end{array}$ & $\begin{array}{l}\text { Política/ Estrategia de enfrenta- } \\
\text { miento } \\
\text { Comunicación de propagación } \\
\text { y presencia "pirata” en el espacio } \\
\text { público }\end{array}$ & $\begin{array}{l}\text { Estrategia del enfrentamiento } \\
\text { externo } \\
\text { Identidad colectiva "militante" }\end{array}$ \\
\hline $\begin{array}{l}\text { Combrailles } \\
\text { Durables } \\
\text { "velada" }\end{array}$ & $\begin{array}{l}\text { Deliberativa } \\
\text { Encuentros cotidianos } \\
\text { Utopía colectiva }\end{array}$ & $\begin{array}{l}\text { Política/ Estrategia "velada" } \\
\text { Comunicación de propagación y } \\
\text { presencia "velada" en el espacio } \\
\text { público }\end{array}$ & $\begin{array}{l}\text { El riesgo esquizofrénico } \\
\text { Identidad colectiva "militante" y } \\
\text { "velada" externamente }\end{array}$ \\
\hline
\end{tabular}

Nota. Elaboración propia. 
activismo utópico espantoso desde una perspectiva institucional, y el utilitarismo destructivo para el potencial de transformación social en el germen de sus acciones (tabla 3). Este riesgo es muy a menudo subrayado por los actores "velados" que tienen miedo de "perder su alma"."1

A través del prisma del cambio institucional, una transformación gradual de los hábitos de hacer y pensar (Veblen, 1970), hemos tratado de cuestionar las prácticas de comunicación de los actores socialmente innovadores en la Ess. La contribución importante sigue siendo la aparición de actores "velados" con una voluntad de transformación simbolizada por prácticas estratégicas de comunicación ocultas detrás de una aparente normatividad de los protagonistas con altos bagajes socioculturales. El avance descrito aquí tiene una tendencia a desafiar las dicotomías establecidas en relación con la Ess (economía social isomorfa vs. economía solidaria transformadora), y la innovación social (una versión "débil” vs. una "versión fuerte"). De hecho, los procesos que se activan en estos dos campos son mucho más complejos y mucho más ambivalentes. El enfoque comunicacional muestra el camino en esta dirección pero sería importante ir más allá y romper la dicotomía de la comunicación de las estructuras de la Ess (marketing vs. política) y también la pareja habermasiana de la comunicación política (comunicación estratégica vs. normativa). Entonces, parece que el trabajo de los investigadores de la Escuela de Minas de París (Akrich, Callon y Latour, 2006) sobre el proceso estratégico de la traducción puede ser movilizado no solamente para mejorar el entendimiento del proceso de las negociaciones y de los actos de comunicación que constantemente se producen en el corazón de las estructuras portadoras de innovación social, sino también en la interfaz entre este y las instituciones sociales.

\section{Referencias}

Akrich, M., Callon, M. y Latour, B. (2006). Sociologie de la traduction - Textes fondateurs. Paris: Ecole des Mines.

Besançon, E. (2014). Théories et pratiques du changement institutionnel en économie solidaire. Une approche institutionnaliste par l'innovation sociale. Amiens.

Besançon, E., Chochoy, N. y Guyon, T. (2013). L'innovation sociale, principes et fondements d'un concept. Lesprit Economique. L'Harmattan, Coll.

11 Palabras de uno de los integrantes de la scic Combrailles Durables.
Besançon, E. y Chochoy, N. (2014). Les Marqueurs d'Innovation sociale : un exemple de recherche partenariale contribuant à l'amorce de nouvelles trajectoires d'innovation. En 4ème Colloque international du crises, La transformation sociale par l'innovation sociale: crises.

Bouchard, M. J. (2011). Léconomie sociale vecteur d'innovation. L'expérience du Québec. Quebec: Presses de l'Université du Québec.

Burawoy, M. (2003). Létude de cas élargie. Une approche réflexive, historique et comparée de l'enquête de terrain. En D. Céfai, L'enquête terrain. Paris: La Découverte.

Cary, P. y Laville, J. L. (2015). Léconomie solidaire : entre transformations institutionelles et chantiers théoriques. Revue française de socio-économie, 15, 23-37.

Céfai, D. (2003). Lénquête de terrain. Paris: La Découverte.

Chambon, J. L., David A. y Devevey J. M. (1982). Les innovations sociales. París : Presses Universitaires de France.

Chirouze, Y. (2007). Le marketing. Etudes et stratégies (2ème éd.). Paris.

Cloutier, J. (2003). Qu'est ce que l'innovation sociale? $\mathrm{Ca}$ hiers du crises, Collection Études Théoriques ET0314.

Dacheux, E. (2011a). Principes d'économie solidaire. Paris: Ellipses.

Dacheux, E. (2011b). Economie solidaire et communication. Hermes (CNRS), 61, 131-144.

Dacheux, E. (dir.). (2007). Communiquer l'utopie: economie solidaire et démocratie. Paris : L'Harmattan.

Dacheux, E. (2004). La communication : éléments de synthèse. Commun. langages, 141(1), 61-70. doi: http:// dx.doi.org/10.3406/colan.2004.3288

Dacheux, E. (1994). Les stratégies de communication persuasive dasn l'Union européenne. Paris: L'Harmattan.

Decaudin, J. M. (1999). La communication marketing. Paris : Economica.

Dilliere-Brooks, S. (2007). Entretien avec Eric Dacheux. Communication des organisations, 31, 267-273.

Dimaggio, P. y Powell, W. (1983). The iron cage revisited: institutional isomorphism and collective rationality in organisational fiels. American Sociological Review, 48(2), 147-162.

Draperi, J. F. (2013). Rendre possible un autre monde: Economie sociales, coopératives et développement durable. Montreuil: Presses de l'économie sociale.

Durance, P. (2011). L'innovation sociale, ou les nouvelles voix $d u$ changement. Paris.

Eme, B. y Laville, J. L. (1992). Cohésion Sociale et emploi. Paris: Desclée de Brouwer. 
Frère, B. (2006). Le nouvel esprit solidaire. Desclée de Brouwer.

Habermas, J. (1985). Le discours philosophique de la modernité. Paris: Gallimard.

Habermas, J. (1987). Théorie de l'agir communicationnel (Tome 1 \& 2). Paris: Fayard.

Hiez, D. y Lavillunière, E. (2013). Vers une théorie de léconomie sociale et solidaire, Coll. Droit et économie sociale et solidaire. Larcier.

Klein, J. L., Laville, J. L. y Moulaert, F. (2014). L'innovation sociale. Toulouse: Eres.

Laville, J. L. y Cattani, A. D. (2005). Dictionnaire de l'autre économie. Paris: Desclée de Brouwer.

Laville J. L. y Sainsaulieu, R. (2013). Lassociation - Sociologie et économie. Paris: Pluriel.

Laville, J. L. (1994). Léconomie solidaire une perspective internationale. Paris: Desclée de Bouwer

Laville J. L. (coord.). (2011). Léconomie solidaire. Coll. Les essentiels d'Hermès.

Lefort, C. (1986). Essais sur le politique : XIXE et XXE siècles. Paris : Editions du Seuil.

Lévesque, B. (2002). Les entreprises d'économie sociale, plus porteuses d'innovations sociales que les autres? Cah. du Cris. Vol. et0205.

Lévesque B. (2007). Le potentiel d'innovation sociale de l'économie sociale : quelques éléments de problématique. Econ. Solidar, 38(1),13-48.

Lindon, D. (1991). Le marketing. Paris: Nathan.

Mucchielli, A. (2000). La nouvelle communication. Paris: Armand Colin.

OCDE (2005). Guidelines for collecting and Interpreting Innovation Data. Recuperado de http://www.oecd.org/innovation/inno/oslomanualguidelinesforcollectingand interpretinginnovationdata3rdedition.htm
Peck, J. (2013). Social innovation... at the limits of neoliberalism. En Klein, J. L. y Roy, M, Pour un nouveau monde le défi d'innover (pp. 1130). Quebec: Presses de l'Université du Québec.

Polanyi, K. (1974). Personal knowledge : towards a post-critical philosophy. Chicago: University of Chicago Press.

Polanyi, K. (1983). La grande transformation. Paris: Gallimard.

Polanyi, K. (2007). Le sophisme économiste. La revue du Mauss, 29, 63-79.

Quéré, L. (1991). D’un modèle épistémologique de la communication à un modèle praxéologique. Réseaux, 9 , 6-47, 69-90.

Richez-Battesti, N. (2008). Innovations sociales et territoires: une analyse en termes de proximité. Une illustration par les banques coopératives. En Zaoual, H. (dir.), Développement durable des territoires, économie sociale, environnement et innovation (pp. 6187). Paris: L'Harmattan.

Schumpeter, J. A. (1939). A Theoretical, Historical and Statistical Analysis of the Capitalist Process. New York: McGraw-Hill Compagny.

Shannon, C. y Weaver, W. (1975). Théorie mathématique de la communication. Retz-depl.

Taylor, J. B. (1970). Introducing social innovation. Journal of applied behavior sciences, 6(1), 66-77.

Varela, F. J. (1989). Connaître les sciences cognitives, tendances et perspectives. Paris: Le Seuil.

Veblen, T. (1970). Théorie de la classe de loisir. Paris: Gallimard.

Wolton, D. (1989). La communication politique: construction d'un modèle. Hermes (CNRS), 4, 27-41. 